\title{
Research on the Optimized Uprooted Speed Model of Cassava Root
}

\author{
Wang Yang ${ }^{1, a}$, Jian Yang ${ }^{1, b}$, Jiaqi Zhao ${ }^{1, c}$, Zhaoxin Liang ${ }^{2, c}$, Jianlin Mo $^{2, c}$ \\ ${ }^{1}$ College of Mechanical Engineering, Guangxi University, Nanning 530004, China \\ ${ }^{2}$ Guangxi Research Institute of Agricultural Machinery, Guangxi University, \\ Nanning 530001, China \\ ayanghope@163.com, byangokok@gxu.edu.cn, cyangokok@gxu.edu.cn
}

Keywords: Cassava root, Uprooted speed model, Optimized, Harvesting machinery

\begin{abstract}
In order to obtain the optimized manual uprooted speed model of cassava root and the mechanical optimized uprooted speed model, the acceleration curves of cassava root uprooting of the experienced farmers are tested and the changing laws of the curves are studied by statistical analysis and nonlinear fitting method. Then, the optimized manual uprooted speed model is selected by the laws and based on it the mechanical optimized uprooted speed model is established. The results show that the optimized manual uprooted speed model is high nonlinearity, but root dithering accompanies the process of uprooting. The speed variation curves can be superimposed by line and sine curve or concave downward parabola and sine curve. The latter is selected as the form of the mechanical optimized uprooted speed model for reducing power consumption and harvest losses.
\end{abstract}

\section{Introduction}

In foreign countries, lots of researches on cassava root harvesting machinery have been carried out and gained some achievements[1-3], but it is still in its infancy in China. Dig-uprooting is the main method for cassava root harvest because of its excellent adaptability for various growth situations and soil, not being easy to damage the root and low consumption. The key issue of the method is to determine the optimized uprooted speed model. According to the test of uprooting the root manually, when the root is uprooted by experienced farmers, there would be lower losses and higher efficiency and vice versa. Test results shows that customary harvest experience plays an important role in reducing havest losses and improving harvest efficiency. So in this paper, the acceleration curves of cassava root uprooting of the experienced farmers are tested and the changing laws of the curves are studied by statistical analysis and nonlinear fitting method. Then, the optimized manual uprooted speed model is selected by the laws and based on it the mechanical optimized uprooted speed model is established.

\section{The Determination of the Manual Uprooted Acceleration of Cassava Root}

Test Method.The changing laws of uprooted speed are different for different people. For the experienced farmers, there would be lower losses and higher efficiency. In order to obtain the optimized manual uprooted speed model of cassava root and reduce the cost and time, the typical sampling method is used in the test. The acceleration curves of cassava root uprooting of ten farmers with thirty years harvesting experience are tested. Fifteen cassavas are uprooted randomly by each farmer and then, the loss and damage of the root are measured.

Test Results and Analysis.Finally,150 acceleration curves are obtained. Meanwhile, loss and damage of the root are measured. According to the observation and statistical analysis of the curves, the results show that the variation of $63 \%$ of the acceleration curves is similar, as shown in figure 2 .

In figure 2, before the maximum acceleration appears, there is a similar variation among them: acceleration increases presenting bell month pattern in the curves along with time. During this period, the soil is separated by uprooted root and root would be broken off easily because root stress is too large. After the maximum acceleration appears, the variations of acceleration curves vary 
greatly. In this stage soil and cassava separated in dithering and root would not be broken off easily because root stress is small. Thus, this paper focuses on the variation of acceleration before the maximum acceleration appears. Due to the differences in the growth situation of root and the experience and physical strength of farmers, the acceleration variation and uprooting time are different. Thus, in order to select the optimized manual uprooted speed model, an optimal acceleration curve (the shortest uprooting time and no root loss) is selected from each farmer's acceleration curves as research object.

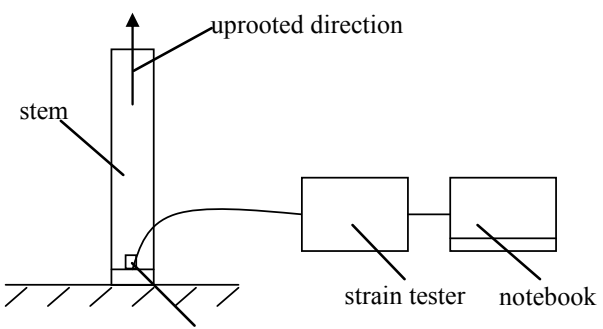

acceleration sensor

Fig.1 Determination of the acceleration

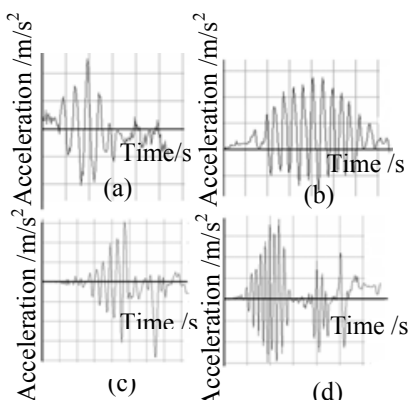

Fig.2 Acceleration curves of uprooting of cassava root manually

Acceleration Curves Fitting and Analysis.Using nonlinear fitting method[4], the corresponding acceleration mathematical model was obtained after fitting the ten optimal acceleration curves, as shown from equation (1) to equation (10). The results of the model test shows that the total variance determination coefficient of time on acceleration is from 0.87 to 0.92 . According to equation from (1) to (10), acceleration and time present complicated highly nonlinear relationship.

$$
\begin{aligned}
\hat{a}_{1}= & 0.364+0.113 t-0.161 t^{2}+0.269 e^{0.762 t} \sin (-21.179 t+64.669) \\
\hat{a}_{2}= & 0.349+3.3 \times 10^{-7} e^{9.678 t} \sin (26.358 t-37.102)+0.311 t^{2} \\
& +0.435 e^{1.347 t} \sin (28.438 t-12) \\
\hat{a}_{3}= & 0.065-0.192 e^{2.013 t} \sin (29.433 t-39)+0.093 t^{2} \\
\hat{a}_{4}= & 0.3+1.1 \times 10^{-18} e^{25.787 t} \sin (-0.071 t-3)+0.14 e^{1.457 t} \sin (18.744 t-32.12) \\
& -0.208 \sin (-13.328 t+8.657) \\
\hat{a}_{5}= & 0.522-3 \times 10^{-6} e^{10.642 t} \sin (-34 t+24.756)-0.027 e^{4.362 t} \cos (-18.515 t+4.078) \\
& -0.99 t^{2}+1.316 \sin (26.668 t) \\
\hat{a}_{6}= & 0.468+0.012 \sin (3.56 t+0.496)+0.512 e^{0.143 t} \sin (23.841 t-42.182) \\
& +0.718 e^{-3.815 t} \sin (21.854 t-1.261) \\
\hat{a}_{7}= & 2572.987-3995.5 \sin (-0.227 t+7.944)+1422.23 \cos (-0.384 t+0.153) \\
& +0.183 e^{1.82 t} \sin (-11.346 t+20.422)-2.072 \sin (-14.41 t-12.676) \\
\hat{a}_{8}= & 0.457+0.292 t^{4}+109.443 \sin (11.989 t-7.68)+109.221 \cos (12 t-6.118) \\
& -0.5 e^{1.1 t} \sin (-21.968 t+3.06)-0.139 e^{3.106 t} \sin (-30.318 t+16.486)-0.871 t \\
\hat{a}_{9}= & 476.357-39.043 t^{4}+0.024 e^{3.473 t} \sin (-40.89 t+50.82)+0.033 e^{3.82 t} \\
& \times \sin (-20.627 t+18.79)-707.55 \sin (-1.57 t-1.303) \\
& -1183.6 \sin (-1.223 t+1.778) \\
\hat{a}_{10}= & 0.14-1.841 \sin (20.65 t-23.27)+1.526 \cos (22.589 t-12.616) \\
& -0.059 e^{3.268 t} \sin (-29.6 t+31.426) \\
&
\end{aligned}
$$


The fitting curves are shown in figure 3 . The solid line is measured value, and the virtual line is fitting expression calculated value. In figure 3, the calculated value is consistent with the measured one, which presents the high precision of the mathematical model. Meanwhile, in figure 3, the uprooting time is different for different farmers due to their physical strength and cassava growth. The shorter time means the higher working efficiency. Therefore, when the optimized manual uprooted acceleration model is obtained, time requires less than 2s. Farmer 2, 3, 4, 5, 8, 9, 10's uprooted time is less than $2 \mathrm{~s}$, and have no root loss and damage. Thus, these models could be the representatives of the optimized manual uprooted acceleration model.
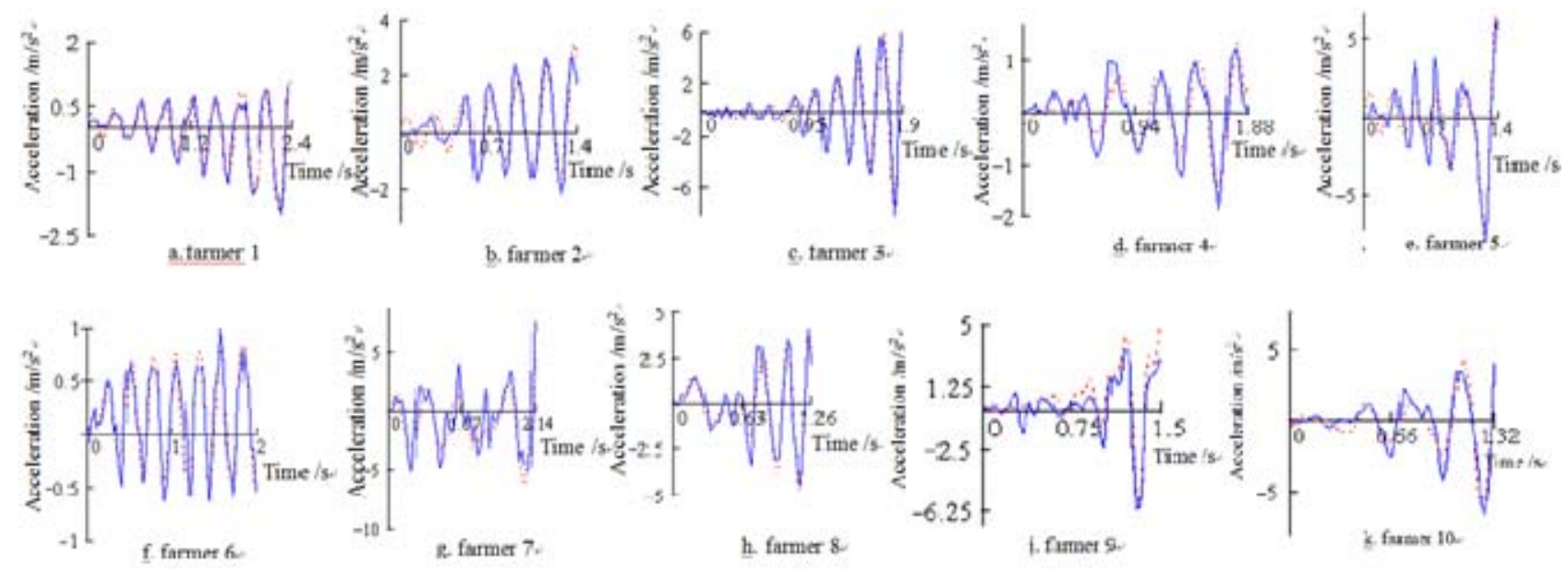

Fig. 3 The comparison of the acceleration curves

\section{The Optimized Manual Uprooted Speed and Analysis}

When $t=0$, uprooted velocity is zero.The optimized manual uprooted speed model is obtained by integrating the equation from (2) to (5) and from (8) to (10). The speed model is shown in the equation from (11) to (17).

$$
\begin{aligned}
v_{2}= & 0.012+0.35 t+0.1 t^{3}+4.18 \times 10^{-10} e^{9.68 t}[9.68 \sin (26.36 t-37.1)-26.36 \cos (26.36 t-37.1)] \\
& +5.67 \times 10^{-4} e^{1.35 t}[1.35 \sin (28.44 t-12)-28.44 \cos (28.44 t-12)] \\
v_{3}= & 2.16 \times 10^{-3}+0.065 t+0.03 t^{3}-2.2 \times 10^{-4} e^{2.01 t}[2.01 \sin (29.43 t-39)-29.43 \cos (29.43 t-39)] \\
v_{4}= & 6.03 \times 10^{-3}+0.3 t-0.016 \cos (-13.33 t+8.66) \\
& +3.96 \times 10^{-4} e^{1.46 t}[1.46 \sin (18.74 t-32.12)-18.74 \cos (18.74 t-32.12)] \\
& +1.65 \times 10^{-21} e^{25.79 t}[25.79 \sin (-0.07 t-3)+0.07 \cos (-0.07 t-3)] \\
v_{5}= & 0.05+0.52 t-0.33 t^{3}-0.05 \cos (26.67 t)-7.46 \times 10^{-5} e^{4.36 t} \\
& \times[4.36 \cos (-18.52 t+4.08)-18.52 \sin (-18.52 t+4.08)] \\
& -2.36 \times 10^{-9} e^{10.64 t}[10.64 \sin (-34 t+24.76)+34 \cos (-34 t+24.76)] \\
v_{8}= & 0.11+0.45 t-0.44 t^{2}+0.06 t^{5}-9.13 \cos (12 t-7.68) \\
& +9.1 \sin (12 t-6.12)-1.03 \times 10^{-3} e^{1.1 t}[1.1 \sin (-21.97 t+3.06)-21.97 \cos (-21.97 t+3.06)] \\
& -1.5 \times 10^{-4} e^{3.11 t}[3.11 \sin (-30.32 t+16.49)-30.32 \cos (-30.32 t+16.49)] \\
v_{9}= & -79.75+476.36 t-7.81 t^{5}-450.67 \cos (-1.57 t-1.3) \\
& -967.79 \cos (-1.22 t+1.78)+1.43 \times 10^{-5} e^{3.47 t} \\
& \times[3.47 \sin (-40.89 t+50.82)+40.89 \cos (-40.89 t+50.82)]+7.5 \times 10^{-5} e^{3.82 t} \\
& \times[3.82 \sin (-20.63 t+18.79)+20.63 \cos (-20.63 t+18.79)]
\end{aligned}
$$




$$
\begin{aligned}
v_{10}= & 0.03+0.14 t+0.09 \cos (20.65 t-23.27)+0.07 \sin (22.59 t-12.62) \\
& -1.22 \times 10^{-4} e^{3.27 t}[3.27 \sin (-21.74 t+24.86)+21.74 \cos (-21.74 t+24.86)]
\end{aligned}
$$

The variations of the optimized manual uprooted speed are obtained by simulated calculation in equation from (11) to (17), as shown in figure 4 . The speed variation can be divided into two types: one is shown in figure 4(a), the other in figure 4b. But figure 4(a) is in a large proportion. According to equation from (11) to (17), the relationship between speed and time is complicated and highly nonlinear, but dithering accompanies the process of uprooting. In figure 4(a), speed variation curves can be superimposed by line and sine curve. The simplified mathematical model is shown in equation (18) .In figure 4(b), speed variation curves can be superimposed by concave downward parabola and sine curve. The simplified mathematical model is shown in equation (19).

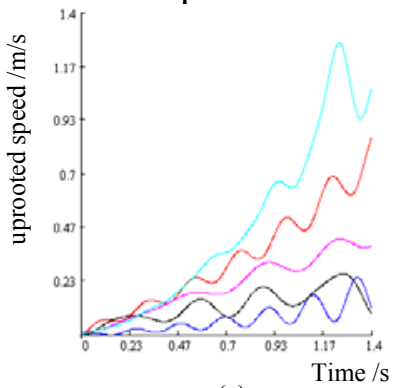

(a)

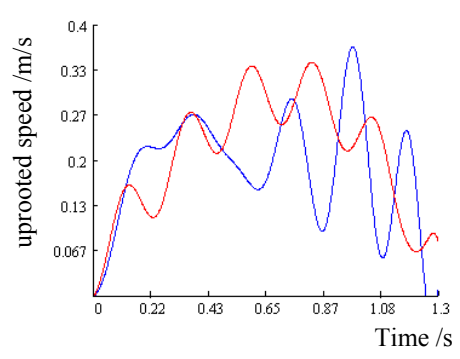

(b)

Fig.4 The optimum uprooted speed of root manually

$$
\begin{aligned}
& v=\left\{\begin{array}{cc}
a_{1} t & 0 \leq t \leq t_{1} \\
a_{1} t+b_{1} \sin \left(2 \pi f_{1} t+\varphi_{1}\right) & t_{1}<t
\end{array}\right. \\
& v=t\left(a_{2}-b_{2} t\right)+c_{2} \sin \left(2 \pi f_{2} t+\varphi_{2}\right)
\end{aligned}
$$

In equations,$a_{1} 、 b_{1} 、 f_{1} 、 \varphi_{1} 、 a_{2} 、 b_{2} 、 c_{2} 、 f_{2} 、 \varphi_{2}$ are constant respectively.

When $a_{1} 、 b_{1} 、 f_{1} 、 \varphi_{1} 、 a_{2} 、 b_{2} 、 c_{2} 、 f_{2} 、 \varphi_{2}$ are the appropriate value, the curves can be drafted by equation (18) and (19), as shown in figure 5. The approximation of speed variation in figure 4(a) and 5(a) and in figure 4(b) and 5(b) shows that the formula (18) and (19) can be in place of the optimized manual uprooted speed model.

\section{Form of the Optimized Uprooted Speed Model of the Uproot Mechanism}

The equation (18) and (19) are the approximate expression of the optimized manual uprooted speed model. The curves can be drafted by the equation (18) and (19), as shown in Figure 5. In Figure 5(a) and 5(b), when $0<t<0.12 \mathrm{~s}$, speed in figure 5(b) is higher than in Figure 5(a). After $t=0.12 \mathrm{~s}$, speed in Figure 5(b) is lower than in Figure 5(a). And speed variation in the process of root uprooting is relatively gentle in figure 5(b). According to the analysis of the uprooting process[5], when the root is uprooted (before the ring shear fracture surface appears), higher speed is beneficial to improving efficiency and avoiding breaking off the root due to the small effective stress. In the stage from maximum uprooted force appearing to soil separation, uprooted speed is small and the variation is relatively gentle, which is beneficial to reducing maximum uprooted force, root effective stress, power consumption and harvest losses. Therefore, equation (19) can be as the form of the optimized uprooted speed model of the uproot mechanism. 


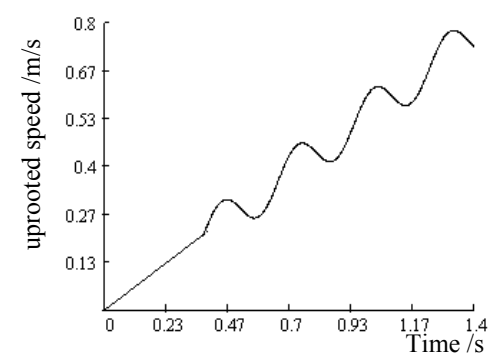

(a)

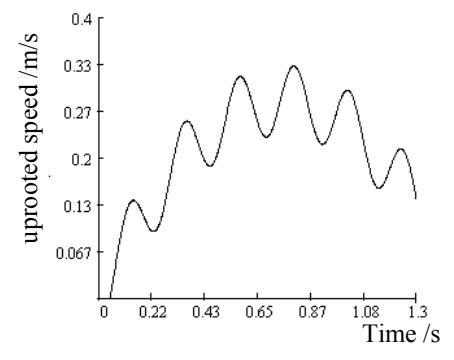

(b)

Fig.5 Simplified of the optimum uprooted speed of root

\section{Conclusions}

(1)The optimized manual uprooted speed model is high nonlinearity, but root dithering accompanies the process of uprooting. The speed variation curves can be divided into two types: one can be superimposed by line and sine curve, the other can be superimposed by concave downward parabola and sine curve.

(2) The speed model superimposed by concave downward parabola and sine curve can be as the form of the optimized uprooted speed model of the uproot mechanism for improving harvest efficiency and reducing maximum uprooted force, root effective stress, power consumption and harvest losses.

\section{Acknowledgements}

This work was financially supported by the National Natural Science Foundation of China (No. 51065003).

\section{References}

[1] P. O Kolawole,L. Agbetoye ,S. A Ogunlowo: Sustainability Vol.2(2010), p.3681-3694.

[2] M. H. H. Razali,H. H. Muhammad,N. A. Mohd,et al: Journal of Crop Science Vol.2 (2011), p.21-26.

[3] L.A.S. Agbetoye, J. Dyson, J. Kilgour:Journal of Agricultural Engineering Research Vol. 75 (2000),p.39-48.

[4] W.D. Ru: SPSS for Windows Statistical Analysis(Publishing House of Electronics Industry, Beijing 2006).

[5] W. Yang, G.W. Cai, J. Yang, et al: Applied Mechanics and Materials, Vols.215-216 (2012), p.1122-1125. 